\title{
Black hole and fuzzy objects in the BFSS matrix model
}

\author{
Yoshifumi Hyakutake \\ College of Science, Ibaraki University, Bunkyo 2-1-1, Mito, Ibaraki 310-8512, Japan
}

(Received 3 August 2018; published 30 August 2018)

\begin{abstract}
We conjecture fuzzy configurations of D0-branes in the Banks-Fischler-Shenker-Susskind (BFSS) matrix model as microstates of the black hole. Fuzzy configurations of D0-branes consist of localized fuzzy objects in three spatial directions which are smeared into six internal directions. Since the solutions are time dependent, these are non-Bogomol'nyi-Prasad-Sommerfield (non-BPS) configurations and have internal energy compared with static case. Especially, we examine the smeared fuzzy sphere in the BFSS matrix model, which will correspond to the microstate of the charged black hole in four dimensions, and compare the effective potential in that background with the result obtained by the near horizon geometry of the black 0 -brane. The qualitative features of two descriptions agree with each other; thus, we expect that the smeared fuzzy sphere corresponds to one of the microstates of the charged black hole. We also examine the smeared fuzzy cylinder which will correspond to the flat space time.
\end{abstract}

DOI: 10.1103/PhysRevD.98.046023

\section{INTRODUCTION}

Superstring theory is the promising candidate for the theory of quantum gravity. In the superstring theory, D-branes play important roles in investigating both gauge theory and gravity theory [1]. Some black holes are realized as bound states of D-branes, and microstates of the black hole are constructed from the gauge theory on the D-branes [2]. This shows that the quantum nature of the gravity can be captured by analyzing corresponding quantum field theory.

Actually, quantum aspects of black holes are investigated considerably via matrix models, which are the nonperturbative formulation of superstring theories [3-6]. In 1996, Banks, Fischler, Shenker, and Susskind proposed a nonperturbative formulation of M-theory (BFSS matrix model) [3]. This theory is obtained by dimensional reduction of 10 dimensional super Yang-Mills theory to quantum mechanics, which is identified with the effective action for multiple D0-branes [7]. It is remarkable that although the BFSS matrix model is the quantum mechanical system, it can reproduce the gravitational force between two D0-branes $[3,8-10] .{ }^{1}$ Hence, the BFSS matrix model captures the nature of the gravity, and it is possible to investigate black hole physics in detail.

\footnotetext{
${ }^{1}$ Originally BFSS proposed to make the size of matrices infinite. In Ref. [11], the finite case was proposed as discretized light cone quantization of M-theory. See Ref. [12], for example, for a review of the BFSS matrix model.

Published by the American Physical Society under the terms of the Creative Commons Attribution 4.0 International license. Further distribution of this work must maintain attribution to the author(s) and the published article's title, journal citation, and DOI. Funded by SCOAP ${ }^{3}$.
}

In fact, there are several works which construct the Schwarzschild black hole in various dimensions from the BFSS matrix model [13-17]. The thermodynamics of the black hole is reproduced qualitatively from the BFSS matrix model in Refs. [13-16], and the instability of black string is examined in Ref. [17]. Especially, a fuzzy sphere configuration of D0-branes is used to describe the black hole in Ref. [18]. In that paper, the effective potential for a test D0-brane in the background of the fuzzy sphere is evaluated at one-loop level, and it agrees with the result of the gravity side qualitatively.

In 1997, Maldacena conjectured the gauge/gravity correspondence [19], and it is confirmed that physical quantities in the gauge theory, such as correlation functions, are consistently calculated from the gravity side [20,21]. From the viewpoint of this conjecture, it is natural to regard that the BFSS matrix model corresponds to the near horizon geometry of black 0-brane [22]. Since the gauge/gravity correspondence is a kind of weak/strong coupling duality, it is hard to test the conjecture analytically. However, recently there have been several numerical tests of this conjecture for the thermal system of D0-branes [23-29]. Especially, the numerical study for the black hole geometry is considered in Ref. [30].

If the gauge/gravity correspondence is correct even for the nonsupersymmetric system, it is important to construct the black hole geometry in the BFSS matrix model. In Ref. [31], a smeared black 0-brane solution and its thermodynamic properties are investigated. The black 0-brane is smeared along six internal directions, so it becomes a charged black hole in four dimensions. Then it is interesting to consider corresponding configurations in the BFSS matrix model. In this paper, we conjecture that fuzzy configurations of 
D0-branes, which are time dependent fuzzy objects smeared along six internal directions, correspond to the microstates of the smeared black 0-brane in the near horizon limit. These fuzzy objects are bound states of D0-branes and are oscillating around the origin of three spatial directions.

The organization of this paper is as follows. In Sec. II, we review the BFSS matrix model and construct time dependent fuzzy objects, including the fuzzy sphere and fuzzy cylinder. In Sec. III, we review the one-loop effective potential for the test D0-brane in the background of fuzzy configuration. In Sec. IV, the effective potentials between fuzzy objects, such as the fuzzy sphere and cylinder, and the test D0-brane are calculated explicitly. We compare properties of these effective potentials with the result obtained by the gravity side in Sec. V. Section VI is devoted to conclusion and discussion.

\section{TIME DEPENDENT FUZZY OBJECTS IN THE BFSS MATRIX MODEL}

In this section, we consider time dependent configurations of $N$ D0-branes in the BFSS matrix model, which will correspond to the microstates of the black hole. Since we are interested in the black hole in four dimensional spacetime, we make a fuzzy object in three spatial directions via D0-branes and smear it along the remaining six internal directions. The equations of motion for the fuzzy object are expressed by simultaneous nonlinear differential equations and solutions have nontrivial time dependence in general. We show the numerical plot for the $N=2$ case which represents a nontrivial bound state of two D0-branes, and then explains the analytic solutions for oscillating the fuzzy sphere and fuzzy cylinder.

Let us consider the BFSS matrix model which describes the dynamics of multiple D0-branes [3]. The action for D0-branes can be obtained by the dimensional reduction of 10 dimensional $\mathcal{N}=1$ super Yang-Mills theory to 1 dimensional super quantum mechanics [7]. The supermultiplet consists of a gauge field $A_{t}, 9$ scalar fields $\Phi_{i}$ and a Majorana-Weyl fermion $\theta$. These are expressed by $N \times N$ matrices, and the action for multiple D0-branes is given by

$$
\begin{aligned}
\mathcal{S}_{0}= & \frac{1}{g_{\mathrm{YM}}^{2}} \int d t \operatorname{tr}\left(\frac{1}{2} D_{t} \Phi_{i} D_{t} \Phi^{i}+\frac{1}{4}\left[\Phi_{i}, \Phi_{j}\right]^{2}\right. \\
& \left.+\frac{i}{2} \theta^{T} D_{t} \theta+\frac{1}{2} \theta^{T} \gamma^{i}\left[\Phi_{i}, \theta\right]\right),
\end{aligned}
$$

where $i, j=1, \ldots, 9, D_{t}=\partial_{t}-i\left[A_{t},\right]$ and $\gamma^{i}$ are $16 \times 16$ matrices. The coupling constant $g_{\mathrm{YM}}$ has mass dimension $3 / 2$. Note that there are 9 scalar fields $X_{i}=2 \pi \ell_{s}^{2} \Phi_{i}$, and the diagonal element of $X_{i}$ corresponds to a position of each D0-brane in the $x_{i}$ direction. Thus, the size of the matrices $N$ is equal to the number of D0-branes. By setting $\theta=0$, the equations of motion for $\Phi^{i}$ and $A_{t}$ become

$$
D_{t}\left(D_{t} \Phi_{i}\right)=\left[\Phi^{j},\left[\Phi_{i}, \Phi_{j}\right]\right], \quad\left[\Phi^{i}, D_{t} \Phi_{i}\right]=0 .
$$

The equation of motion for $\theta$ is trivially satisfied when $\theta=0$.

Let us examine Eq. (2) to construct the fuzzy object which would correspond to the microstate of the black hole in four dimensions. First of all, we set $A_{t}=0$ and choose $\Phi_{a}(a=1,2,3)$ as

$$
\begin{aligned}
& \left(\Phi_{1}^{\mathrm{bg}}\right)_{m n}=\frac{1}{2} \rho_{m}(t) \delta_{m+1, n}+\frac{1}{2} \rho_{n}(t) \delta_{m, n+1} \\
& \left(\Phi_{2}^{\mathrm{bg}}\right)_{m n}=-\frac{i}{2} \rho_{m}(t) \delta_{m+1, n}+\frac{i}{2} \rho_{n}(t) \delta_{m, n+1} \\
& \left(\Phi_{3}^{\mathrm{bg}}\right)_{m n}=z_{m}(t) \delta_{m, n} .
\end{aligned}
$$

Here $\rho_{m}(t)$ and $z_{m}(t)$ are functions of temporal coordinate $t$, and $m, n=1, \ldots, N$. The superscript bg stands for the background. This ansatz represents the fuzzy object in 3 dimensions which is axially symmetric around the $x^{3}$ direction [32,33]. Roughly speaking, $\rho_{m}$ represents the extension of the fuzzy object from the origin on the $x_{3}=z_{m}$ plane. Therefore, the fuzzy object makes an axially symmetric surface in three dimensions, and it carries a dielectric D2-brane charge. The remaining six scalars $\Phi_{u}(u=4, \ldots, 9)$ are chosen to be diagonal so that the fuzzy object is smeared along six spatial directions.

Let us substitute the ansatz (3) into the equations of motion (2). The differential equations for $\rho_{m}$ and $z_{m}$ are written as

$$
\begin{aligned}
\ddot{\rho}_{m} & =\left\{\frac{1}{2}\left(\rho_{m+1}^{2}-2 \rho_{m}^{2}+\rho_{m-1}^{2}\right)-\left(z_{m+1}-z_{m}\right)^{2}\right\} \rho_{m}, \\
(m & =1, \ldots, N-1) \\
\ddot{z}_{m} & =\rho_{m}^{2}\left(z_{m+1}-z_{m}\right)-\rho_{m-1}^{2}\left(z_{m}-z_{m-1}\right), \\
(m & =1, \ldots, N)
\end{aligned}
$$

where $\rho_{0}=\rho_{N}=z_{0}=z_{N+1}=0$. Note that the second equation in Eq. (2) is automatically satisfied. Since the differential equations (4) are nonlinear, in general, it is impossible to obtain analytic solutions. From the energy conservation, however, we see that

$$
\begin{aligned}
E= & \frac{1}{g_{\mathrm{YM}}^{2}} \operatorname{tr}\left(\frac{1}{2} \dot{\Phi}_{i} \dot{\Phi}^{i}-\frac{1}{4}\left[\Phi_{i}, \Phi_{j}\right]^{2}\right) \\
= & \frac{1}{g_{\mathrm{YM}}^{2}} \sum_{m=1}^{N}\left\{\frac{1}{2}\left(\dot{\rho}_{m}\right)^{2}+\frac{1}{2}\left(\dot{z}_{m}\right)^{2}+\frac{1}{8}\left(\rho_{m}^{2}-\rho_{m-1}^{2}\right)^{2}\right. \\
& \left.+\frac{1}{2}\left(z_{m+1}-z_{m}\right)^{2} \rho_{m}^{2}\right\} .
\end{aligned}
$$

This shows that the ranges of $\rho_{m}$ are finite, and those of $\left(z_{m}-z_{m-1}\right)$ are also finite if $\rho_{m} \neq 0$ for all $m$. If $\rho_{m}=0$ for some $m$, the representation is reducible and it represents 
two or more fuzzy objects. Since separations of those fuzzy objects should be finite, again $\left(z_{m}-z_{m-1}\right)$ are also finite. From the second equation of (4), we note that $\sum_{m=1}^{N} \ddot{z}_{m}=0$, so we set the center of mass $\sum_{m=1}^{N} z_{m}=0$ without loss of generality. Thus, the D0-branes are bounded around the origin in three spatial directions.

Now let us examine three types of solutions of Eq. (4). The first example is the case of $N=2$. Here we set $z_{2}=-z_{1}$. Then differential equations for $\rho_{1}$ and $z_{1}$ are written as

$$
\ddot{\rho}_{1}=-\left(\rho_{1}^{2}+4 z_{1}^{2}\right) \rho_{1}, \quad \ddot{z}_{1}=-2 \rho_{1}^{2} z_{1},
$$

and the energy is given by

$$
E_{N=2}=\frac{1}{g_{\mathrm{YM}}^{2}}\left\{\frac{1}{2}\left(\dot{\rho}_{1}\right)^{2}+\left(\dot{z}_{1}\right)^{2}+\frac{1}{4} \rho_{1}^{4}+2 z_{1}^{2} \rho_{1}^{2}\right\} .
$$

Since $\rho_{1}=0$ corresponds to freely moving two D0-branes, we ignore this case. Then $\rho_{1}$ and $z_{1}$ interact in a nontrivial way, but two D0-branes make a bound state around the origin in three dimensions. The plots of $\rho_{1}(t)$ and $z_{1}(t)$ are shown in Fig. 1. This shows that the dynamics of the fuzzy object is complicated even for $N=2$.

The second example is a fuzzy sphere which oscillates around the origin in three dimensions. The explicit forms of 3 scalar fields $\Phi_{a}^{\mathrm{bg}}$ are given by Eq. (3) with

$$
\rho_{m}=\tilde{r}(t) \sqrt{m(N-m)}, \quad z_{m}=\frac{\tilde{r}(t)}{2}(N-2 m+1) .
$$

We also set $A_{t}^{\mathrm{bg}}=0$ and six scalar fields $\Phi_{u}^{\mathrm{bg}}$ to be diagonal. $|\tilde{r}(t)|$ corresponds to the size of the fuzzy sphere, and the sign of $\tilde{r}(t)$ is related to the orientation of the sphere. The tilde is used to clarify that the quantity has mass dimension. Note that $\Phi_{a}^{\mathrm{bg}}$ can be expressed as [34]

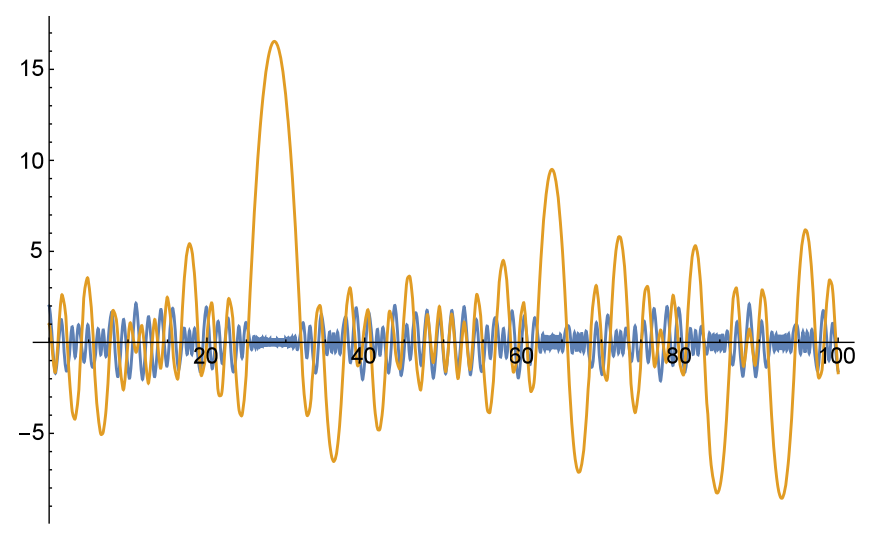

FIG. 1. Plots of $\rho_{1}(t)$ (blue) and $z_{1}(t)$ (yellow). Initial conditions are chosen as $\rho_{1}(0)=z_{1}(0)=1$ and $\dot{\rho}_{1}(0)=\dot{z}_{1}(0)=0$.

$$
\Phi_{a}^{\mathrm{bg}}=\tilde{r}(t) \frac{\Sigma_{a}}{2}, \quad\left[\frac{\Sigma_{a}}{2}, \frac{\Sigma_{b}}{2}\right]=i \epsilon_{a b c} \frac{\Sigma^{c}}{2}
$$

where $a, b, c=1,2,3 . \Sigma_{a} / 2$ are $N$ dimensional irreducible representations of $\mathrm{SU}(2)$ Lie algebra, which satisfy $\Sigma_{1}^{2}+\Sigma_{2}^{2}+\Sigma_{3}^{2}=\left(N^{2}-1\right) \mathbf{1}_{N}$. Inserting Eq. (8) into the equations of motion (4), we obtain a simple equation for $\tilde{r}(t)$ as follows:

$$
\ddot{\tilde{r}}=-2 \tilde{r}^{3}
$$

The above equation corresponds to the classical motion for a particle which is periodically moving in the quartic potential [35]. And the solution is described by using Jacobi's elliptic function sn as

$$
\tilde{r}(t)=c_{1} \operatorname{sn}\left(c_{1} t+c_{2},-1\right),
$$

where $c_{1}(>0)$ and $c_{2}$ are integral constants. $\tilde{r}(t)$ oscillates between $-c_{1}$ and $c_{1}$. The radius of the fuzzy sphere is estimated as

$R_{\mathrm{sph}}=\sqrt{\frac{1}{N} \operatorname{tr}\left(X_{1}^{2}+X_{2}^{2}+X_{3}^{2}\right)}=\pi \ell_{s}^{2} \tilde{r}(t) \sqrt{N^{2}-1}$,

and the maximum value of the radius is given by $\pi \ell_{s}^{2} c_{1} \sqrt{N^{2}-1}$. In other words, $c_{1}$ is linearly related to the size of the fuzzy sphere. Another constant $c_{2}$ can be fixed so that the radius of the fuzzy sphere becomes maximum at $t=0$. The energy of the fuzzy sphere is estimated as

$$
E_{\mathrm{sph}}=\frac{N\left(N^{2}-1\right)}{8 g_{\mathrm{YM}}^{2}}\left(\dot{\tilde{r}}^{2}+\tilde{r}^{4}\right)=\frac{N\left(N^{2}-1\right)}{8 g_{\mathrm{YM}}^{2}} c_{1}^{4} .
$$

Note that this is the internal energy of $N$ D0-branes. If we trust the classical solution naively, the fuzzy sphere oscillates around the origin in three dimensions. And it carries a dielectric D2-brane or anti-D2-brane charge, depending on the orientation of the sphere. In actuality, the fuzzy sphere interacts with the closed strings and it will lose the internal energy during the oscillation.

The third example is an oscillating fuzzy cylinder. The fuzzy cylinder is homogeneously extending along the $x_{3}$ axis and its circular cross section is oscillating on the $\left(x_{1}, x_{2}\right)$ plane. Since the length of the fuzzy cylinder is infinite, the size of the matrices $N$ should be infinite. The explicit forms of three scalar fields $\Phi_{a}^{\mathrm{bg}}$ are given by Eq. (3) with

$$
\rho_{m}=\tilde{\rho}(t), \quad z_{m}=-\tilde{l} m,
$$

where $m$ takes the integer value and $\tilde{l}$ is a typical mass scale of the fuzzy cylinder. In the matrix representation, $\Phi_{a}^{\mathrm{bg}}$ are expressed as 


$$
\Phi_{1}^{\mathrm{bg}}=\tilde{\rho}(t) \Xi_{1}, \quad \Phi_{2}^{\mathrm{bg}}=\tilde{\rho}(t) \Xi_{2}, \quad \Phi_{3}^{\mathrm{bg}}=\tilde{l} \Xi_{3},
$$

$\left[\Xi_{1}, \Xi_{2}\right]=0, \quad\left[\Xi_{2}, \Xi_{3}\right]=i \Xi_{1}, \quad\left[\Xi_{3}, \Xi_{1}\right]=i \Xi_{2}$.

We set $A_{t}^{\mathrm{bg}}=0$ and six scalar fields $\Phi_{u}^{\mathrm{bg}}$ to be diagonal as the case of the fuzzy sphere. Inserting Eq. (14) into the equations of motion (4), we obtain a simple equation for $\tilde{\rho}(t)$ as follows:

$$
\ddot{\tilde{\rho}}=-\tilde{l}^{2} \tilde{\rho} .
$$

And the solution becomes

$$
\tilde{\rho}(t)=c_{3} \cos \left(\tilde{l} t+c_{4}\right) .
$$

Thus, the fuzzy cylinder shrinks and expands like a harmonic oscillator. The radius of the fuzzy cylinder is estimated as

$$
R_{\text {cyl }}=\sqrt{\frac{1}{N} \operatorname{tr}\left(X_{1}^{2}+X_{2}^{2}\right)}=2 \pi \ell_{s}^{2} \tilde{\rho}(t),
$$

and the internal energy is given by

$$
E_{\mathrm{cyl}}=\frac{N}{2 g_{\mathrm{YM}}^{2}}\left(\dot{\tilde{\rho}}^{2}+\tilde{l}^{2} \tilde{\rho}^{2}\right)=\frac{N}{2 g_{\mathrm{YM}}^{2}} \tilde{l}^{2} c_{3}^{2} .
$$

The fuzzy cylinder oscillates around the $x^{3}$ axis with carrying a dielectric D2-brane or anti-D2-brane charge. In actuality, the fuzzy cylinder interacts with the closed strings and it will lose the internal energy during the oscillation.

\section{FLUCTUATIONS AROUND SMEARED FUZZY BACKGROUND}

In the previous section, we have constructed the fuzzy objects, such as fuzzy sphere and cylinder, in three dimensions. In order to identify the fuzzy object with the microstate of the black hole in four dimensions, we need to smear it into spatial $x_{u}(u=4, \ldots, 9)$ directions. Thus, we compactify $x_{u}$ with radius $R_{u}$, and put a copy of the fuzzy object on each position of $\left(x_{4}, \ldots, x_{9}\right)=\left(2 \pi R_{4} n_{4} / Z_{4}, \ldots, 2 \pi R_{9} n_{9} / Z_{9}\right)$. Here, $Z_{u}$ are some integers and $n_{u}$ runs from 1 to $Z_{u}$, and there are $Z=\prod_{u=4}^{9} Z_{u}$ copies of the fuzzy object. By using Eq. (3), nine scalar fields for the smeared fuzzy object are represented as

$$
\begin{aligned}
& \Phi_{a}=\Phi_{a}^{\mathrm{bg}} \otimes \mathbf{1}_{Z}, \quad a=1,2,3, \\
& \Phi_{u}=\mathbf{1}_{N} \otimes P_{u}, \quad u=4, \ldots, 9 .
\end{aligned}
$$

Here $P_{u}$ are $Z \times Z$ diagonal matrices of the forms,

$$
P_{u}=\mathbf{1}_{Z_{4}} \otimes \cdots \otimes \frac{2 \pi \tilde{R}_{u}}{Z_{u}}\left(\begin{array}{lll}
1 & & \\
& \ddots & \\
& & Z_{u}
\end{array}\right) \otimes \cdots \otimes \mathbf{1}_{Z_{9}}
$$

where $\tilde{R}_{u}=R_{u} /\left(2 \pi \ell_{s}^{2}\right)$. Thus, each diagonal component composes a vector $p_{u}=2 \pi \tilde{R}_{u} n_{u} / Z_{u}$. The fuzzy object is completely smeared when we take $Z_{u} \rightarrow \infty$. The energy of the fuzzy objects are slightly modified due to the presence of $P_{u}$. For examples, the internal energies of the fuzzy sphere and fuzzy cylinder are estimated as

$E_{\mathrm{sph}}=\frac{(N Z)^{2}\left(N^{2}-1\right)}{8 \lambda} c_{1}^{4}, \quad E_{\mathrm{cyl}}=\frac{(N Z)^{2}}{2 \lambda} \tilde{l}^{2} c_{3}^{2}$,

where $\lambda=g_{\mathrm{YM}}^{2} N Z$ is the 't Hooft coupling constant. The number of copies $Z$ goes to infinity, and $N$ is also infinite for the fuzzy cylinder.

Below we closely follow Ref. [18] to evaluate the effective potential between the smeared fuzzy object and a test D0-brane. In order to execute this, we start from the Euclidean action of the BFSS matrix model with the background field method. We decompose the scalar fields as $\Phi_{i}=B_{i}+Y_{i}$. Here $B_{i}$ are background fields and $Y_{i}$ are fluctuations. As was solved in the previous section, backgrounds of the gauge field and the Majorana-Weyl fermion are set to be zero. By adding gauge fixing and ghost terms, the action is given by

$$
\begin{aligned}
\mathcal{S}_{\mathrm{E}}= & \frac{1}{2 g_{\mathrm{YM}}^{2}} \int d \tau \operatorname{tr}\left(D_{\tau} \Phi_{i} D_{\tau} \Phi^{i}-\frac{1}{2}\left[\Phi_{i}, \Phi_{j}\right]^{2}\right. \\
& +\theta^{T} D_{\tau} \theta-\theta^{T} \gamma^{i}\left[\Phi_{i}, \theta\right]+\left(\dot{A}_{\tau}-i\left[B_{i}, Y^{i}\right]\right)^{2} \\
& \left.-i \dot{\bar{C}} D_{\tau} C-\left[B_{i}, \bar{C}\right] D^{i} C\right) .
\end{aligned}
$$

Here $\tau=i t$ is the Euclidean time and dot is the derivative with respect to $\tau$. The explicit expressions for the background fields $B_{i}=\left(B_{a}, B_{u}\right)$ are written as

$$
B_{a}=\left(\begin{array}{cc}
\Phi_{a}^{\mathrm{bg}} \otimes \mathbf{1}_{Z} & 0 \\
0 & \tilde{x}_{a}
\end{array}\right), \quad B_{u}=\left(\begin{array}{cc}
\mathbf{1}_{N} \otimes P_{u} & 0 \\
0 & 0
\end{array}\right) .
$$

The first $N Z \times N Z$ block diagonal represents the smeared fuzzy object. The second $1 \times 1$ component does the test D0-brane, and $x_{a}=\left(2 \pi \ell_{s}^{2}\right) \tilde{x}_{a}$ represents its position in three directions. The tilde for $\tilde{x}_{a}$ is used to clarify that the quantity has mass dimension. Below we assume that the test D0-brane is moving very slowly and it is reasonable to neglect the time dependence of $\tilde{x}_{a}$.

Let us consider fluctuations around the background (24). Since we are interested in the effective potential between the fuzzy object and the test D0-brane, we only introduce the fluctuations of off-diagonal parts, 


$$
\begin{array}{rlr}
A_{\tau} & =\left(\begin{array}{cc}
0 & a(\tau) \\
a(\tau)^{\dagger} & 0
\end{array}\right), & \Phi_{i}=B_{i}+\left(\begin{array}{cc}
0 & \phi_{i}(\tau) \\
\phi_{i}(\tau)^{\dagger} & 0
\end{array}\right), \\
\theta & =\left(\begin{array}{cc}
0 & \psi(\tau) \\
\psi(\tau)^{\dagger} & 0
\end{array}\right), & C=\left(\begin{array}{cc}
0 & c(\tau) \\
c^{\dagger}(\tau) & 0
\end{array}\right), \\
\bar{C} & =\left(\begin{array}{cc}
0 & \bar{c}(\tau) \\
\bar{c}^{\dagger}(\tau) & 0
\end{array}\right) .
\end{array}
$$

We substitute the above ansatz into the Euclidean action (23), and expand it up to the quadratic order of the fluctuations. Then the mass squared terms for 10 bosons $\left(a, \phi_{i}\right)$, the Majorana-Weyl fermion $\theta$ and 2 ghosts $c, \bar{c}$ are obtained as follows [18]:

$\Omega_{\mathrm{b}}^{2}=K^{2} \mathbf{1}_{10}+M_{\mathrm{b}}, \quad \Omega_{\mathrm{f}}^{2}=K^{2} \mathbf{1}_{16}+M_{\mathrm{f}}, \quad \Omega_{\mathrm{g}}^{2}=K^{2} \mathbf{1}_{2}$.

Here the diagonal parts of the mass squared terms have the same structure $K^{2}=K_{i} K^{i}$, which is the $(N Z) \times(N Z)$ matrix. The explicit expressions for $K_{i}=\left(K_{a}, K_{u}\right)$ and $K^{2}$ are given by

$K_{a}=Q_{a} \otimes \mathbf{1}_{Z}, \quad K_{u}=\mathbf{1}_{N} \otimes P_{u}, \quad K^{2}=Q^{2} \otimes \mathbf{1}_{Z}+\mathbf{1}_{N} \otimes P^{2}$,

where

$Q_{a}=\Phi_{a}^{\mathrm{bg}}-\tilde{x}_{a} \mathbf{1}_{N}, \quad Q^{2}=\left(\Phi^{\mathrm{bg}}\right)^{2}+\tilde{x}^{2} \mathbf{1}_{N}-2 \tilde{x}^{a} \Phi_{a}^{\mathrm{bg}}$,

and $\tilde{x}^{2}=\tilde{x}_{a} \tilde{x}^{a},\left(\Phi^{\mathrm{bg}}\right)^{2}=\Phi_{a}^{\mathrm{bg}} \Phi^{\mathrm{bg} a}$ and $P^{2}=P_{u} P^{u}$. On the other hand, off-diagonal parts of the mass squared terms, $M_{\mathrm{b}}$ and $M_{\mathrm{f}}$, are written as

$$
\begin{aligned}
& M_{\mathrm{b}}=2 i\left(\begin{array}{cc}
0 & \dot{K}_{j} \\
-\dot{K}_{i} & -i\left[K_{i}, K_{j}\right]
\end{array}\right) \equiv 2 i\left(\begin{array}{cc}
0 & F_{\tau j} \\
F_{i \tau} & F_{i j}
\end{array}\right), \\
& M_{\mathrm{f}}=\gamma^{i} \dot{K}_{i}+\frac{1}{2} \gamma^{i j}\left[K_{i}, K_{j}\right] \equiv \frac{i}{2} \gamma^{\mu \nu} F_{\mu \nu} .
\end{aligned}
$$

In the second line, we introduced " 10 dimensional" gamma matrices $\gamma^{\mu}(\mu=\tau, 1, \ldots, 9)$ and defined $\gamma^{\tau i} \equiv-i \gamma^{i}$. Note that each $F_{\mu \nu}$ is a $(N Z) \times(N Z)$ matrix.

Now we are ready to evaluate the effective potential at one-loop level. The formula for the effective potential is given by

$$
\begin{aligned}
V_{\text {eff }}= & \operatorname{tr}_{\mathrm{b}}\left(\Omega_{\mathrm{b}}\right)-\frac{1}{2} \operatorname{tr}_{\mathrm{f}}\left(\Omega_{\mathrm{f}}\right)-\operatorname{tr}_{\mathrm{g}}\left(\Omega_{\mathrm{g}}\right) \\
= & -\frac{1}{2 \sqrt{\pi}} \int_{0}^{\infty} \frac{d \ell}{\ell^{3 / 2}} \operatorname{tr}_{\mathrm{b}}\left(e^{-\ell \Omega_{\mathrm{b}}^{2}}\right) \\
& +\frac{1}{4 \sqrt{\pi}} \int_{0}^{\infty} \frac{d \ell}{\ell^{3 / 2}} \operatorname{tr}_{\mathrm{f}}\left(e^{-\ell \Omega_{\mathrm{f}}^{2}}\right) \\
& +\frac{1}{2 \sqrt{\pi}} \int_{0}^{\infty} \frac{d \ell}{\ell^{3 / 2}} \operatorname{tr}_{\mathrm{g}}\left(e^{-\ell \Omega_{\mathrm{g}}^{2}}\right) .
\end{aligned}
$$

Each term in the above can be evaluated perturbatively in the interaction picture. In order to evaluate $e^{-\ell \Omega^{2}}=$ $e^{-\ell\left(K^{2}+M\right)}$, let us define $U(\ell) \equiv e^{\ell K^{2}} e^{-\ell \Omega^{2}}$ and $M(\ell) \equiv$ $e^{\ell K^{2}} M e^{-\ell K^{2}}$. The $U(\ell)$ satisfies a differential equation $\frac{d U(\ell)}{d \ell}=-M(\ell) U(\ell)$, and it can be solved as

$$
\begin{aligned}
U(\ell)= & \mathbf{1}-\int_{0}^{\ell} d \ell_{1} M\left(\ell_{1}\right) \\
& +\int_{0}^{\ell} d \ell_{1} M\left(\ell_{1}\right) \int_{0}^{\ell_{1}} d \ell_{2} M\left(\ell_{2}\right)-\cdots
\end{aligned}
$$

Thus, $e^{-\ell \Omega^{2}}=e^{-\ell K^{2}} U(\ell)$ is expanded as

$$
\begin{aligned}
e^{-\ell \Omega^{2}}= & e^{-\ell K^{2}}-\int_{0}^{\ell} d \ell_{1} e^{-\ell K^{2}} M\left(\ell_{1}\right) \\
& +\int_{0}^{\ell} d \ell_{1} \int_{0}^{\ell_{1}} d \ell_{2} e^{-\ell K^{2}} M\left(\ell_{1}\right) M\left(\ell_{2}\right)-\cdots
\end{aligned}
$$

Now it is possible to evaluate the effective action (30) order by order by employing Eq. (32). After some calculations, we see that terms up to the order of $M^{3}$ vanish because of the underlying supersymmetry. The nontrivial contribution arises from the order of $M^{4}$, and the result is given by $[18,36]$

$$
\begin{aligned}
\left.V_{\mathrm{eff}}\right|_{M^{4}}= & -\frac{1}{2 \sqrt{\pi}} \int_{0}^{\infty} \frac{d \ell}{\ell^{3 / 2}} \int_{0}^{\ell} d \ell_{1} \int_{0}^{\ell_{1}} d \ell_{2} \int_{0}^{\ell_{2}} d \ell_{3} \int_{0}^{\ell_{3}} d \ell_{4} \\
& \times \operatorname{tr}_{(N Z)}\left[e ^ { - \ell K ^ { 2 } } \left\{8 F^{\mu}{ }_{\nu}\left(\ell_{1}\right) F_{\rho}^{\nu}\left(\ell_{2}\right) F_{\sigma}^{\rho}\left(\ell_{3}\right) F_{\mu}^{\sigma}\left(\ell_{4}\right)\right.\right. \\
& +16 F_{\mu \nu}\left(\ell_{1}\right) F^{\mu \rho}\left(\ell_{2}\right) F^{\nu \sigma}\left(\ell_{3}\right) F_{\rho \sigma}\left(\ell_{4}\right) \\
& -4 F_{\mu \nu}\left(\ell_{1}\right) F^{\mu \nu}\left(\ell_{2}\right) F_{\rho \sigma}\left(\ell_{3}\right) F^{\rho \sigma}\left(\ell_{4}\right) \\
& \left.\left.-2 F_{\mu \nu}\left(\ell_{1}\right) F_{\rho \sigma}\left(\ell_{2}\right) F^{\mu \nu}\left(\ell_{3}\right) F^{\rho \sigma}\left(\ell_{4}\right)\right\}\right] \\
F_{\mu \nu}(\ell) & \equiv e^{\ell K^{2}} F_{\mu \nu} e^{-\ell K^{2}}
\end{aligned}
$$

where the trace is taken for the $(N Z) \times(N Z)$ matrix. Furthermore the $(N Z) \times(N Z)$ matrix is decomposed into the product of the $N \times N$ matrix and the $Z \times Z$ matrix. Indeed, $e^{-\ell K^{2}}=e^{-\ell Q^{2}} \otimes e^{-\ell P^{2}}$, and the nonzero component of the field strength is $F_{\alpha \beta}(\ell)=G_{\alpha \beta}(\ell) \otimes \mathbf{1}_{Z}$ with

$$
\begin{aligned}
G_{\alpha \beta}(\ell) & \equiv e^{\ell Q^{2}} G_{\alpha \beta} e^{-\ell Q^{2}}, \\
G_{\alpha \beta} & =\left(\begin{array}{cc}
0 & \dot{Q}_{b} \\
-\dot{Q}_{a} & -i\left[Q_{a}, Q_{b}\right]
\end{array}\right),
\end{aligned}
$$

where $\alpha, \beta=\tau, 1,2,3 . Q_{a}$ is defined in Eq. (28), and we set $\dot{\tilde{x}}_{a}=0$ for the slowly moving test D0-brane. Finally, the effective potential (33) is expressed as 


$$
\begin{aligned}
\left.V_{\mathrm{eff}}\right|_{M^{4}}= & -\frac{1}{2 \sqrt{\pi}} \int_{0}^{\infty} \frac{d \ell}{\ell^{3 / 2}} \int_{0}^{\ell} d \ell_{1} \int_{0}^{\ell_{1}} d \ell_{2} \\
& \times \int_{0}^{\ell_{2}} d \ell_{3} \int_{0}^{\ell_{3}} d \ell_{4} \operatorname{tr}_{Z}\left(e^{-\ell P^{2}}\right) \\
& \times \operatorname{tr}_{N}\left[e ^ { - \ell Q ^ { 2 } } \left\{8 G^{\alpha}{ }_{\beta}\left(\ell_{1}\right) G^{\beta}{ }_{\gamma}\left(\ell_{2}\right) G_{\delta}^{\gamma}\left(\ell_{3}\right) G_{\alpha}^{\delta}\left(\ell_{4}\right)\right.\right. \\
& +16 G_{\alpha \beta}\left(\ell_{1}\right) G^{\alpha \gamma}\left(\ell_{2}\right) G^{\beta \delta}\left(\ell_{3}\right) G_{\gamma \delta}\left(\ell_{4}\right) \\
& -4 G_{\alpha \beta}\left(\ell_{1}\right) G^{\alpha \beta}\left(\ell_{2}\right) G_{\gamma \delta}\left(\ell_{3}\right) G^{\gamma \delta}\left(\ell_{4}\right) \\
& \left.\left.-2 G_{\alpha \beta}\left(\ell_{1}\right) G_{\gamma \delta}\left(\ell_{2}\right) G^{\alpha \beta}\left(\ell_{3}\right) G^{\gamma \delta}\left(\ell_{4}\right)\right\}\right] .
\end{aligned}
$$

Thus, $\operatorname{tr}_{(N Z)}$ is factorized into $\operatorname{tr}_{Z}$ and $\operatorname{tr}_{N}$. If we take the large $Z$ limit with $R_{u}$ fixed, the trace $\operatorname{tr}_{Z}$ is transformed into a Gaussian integral.

$$
\operatorname{tr}_{Z}\left(e^{-\ell P^{2}}\right)=\sum_{n_{4}=1}^{Z_{4}} \cdots \sum_{n_{9}=1}^{Z_{9}} e^{-\ell p_{u}^{2}} \sim \frac{\pi^{3} Z}{2^{6} M_{6}} \frac{1}{\ell^{3}},
$$

where $p_{u}=2 \pi \tilde{R}_{u} n_{u} / Z_{u}, \quad \tilde{R}_{u}=R_{u} /\left(2 \pi \ell_{s}^{2}\right)$ and $M_{6}=$ $\prod_{u=4}^{9} 2 \pi \tilde{R}_{u}$. Note that the dependence on $\tilde{x}_{a}$ appears through $Q^{2}$ in Eq. (35).

\section{EFFECTIVE POTENTIALS VIA SMEARED FUZZY OBJECTS}

Let us calculate the effective potentials (35) between the smeared fuzzy objects and the test D0-brane by using
Eqs. (28), (34), and (36). We assume that the test D0-brane is located on the $x_{3}$ axis without loss of generality. So we set $\tilde{x}=\tilde{x}_{3}$ in this section.

\section{A. Effective potential via smeared fuzzy sphere}

In this subsection, we calculate the effective potential between the smeared fuzzy sphere and the test D0-brane. Then $Q^{2}$ in Eq. (28) is evaluated as

$$
Q^{2}=\left(\frac{N^{2}-1}{4} \tilde{r}^{2}+\tilde{x}^{2}\right) \mathbf{1}_{N}-\tilde{r} \tilde{x} \Sigma_{3},
$$

and the field strength $G_{\alpha \beta}(\ell)$ is given by

$$
G_{\alpha \beta}(\ell)=\left(\begin{array}{cc}
0 & \dot{\tilde{r}} \frac{\Sigma_{b}(\theta)}{2} \\
-\dot{\tilde{r}} \frac{\Sigma_{a}(\theta)}{2} & \tilde{r}^{2} \epsilon_{a b c} \frac{\Sigma^{c}(\theta)}{2}
\end{array}\right) .
$$

Here we defined $\theta \equiv 2 \ell \tilde{r} \tilde{x}$ and $\Sigma_{a}(\theta) \equiv e^{\ell Q^{2}} \Sigma_{a} e^{-\ell Q^{2}}$. And the explicit form of $\Sigma_{a}(\theta)$ is given as follows:

$$
\left(\begin{array}{c}
\Sigma_{1}(\theta) \\
\Sigma_{2}(\theta) \\
\Sigma_{3}(\theta)
\end{array}\right)=\left(\begin{array}{ccc}
\cosh \theta & -i \sinh \theta & 0 \\
i \sinh \theta & \cosh \theta & 0 \\
0 & 0 & 1
\end{array}\right)\left(\begin{array}{c}
\Sigma_{1} \\
\Sigma_{2} \\
\Sigma_{3}
\end{array}\right) .
$$

By inserting the above expressions into Eq. (35), the effective potential at $M^{4}$ order is evaluated as follows:

$$
\begin{aligned}
& \left.V_{\mathrm{eff}}\right|_{M^{4}}=-\frac{1}{2^{8} \sqrt{\pi}} \frac{\pi^{3} Z}{M_{6}} \frac{1}{(2 \tilde{r} \tilde{x})^{1 / 2}} \int_{0}^{\infty} \frac{d \theta}{\theta^{9 / 2}} \int_{0}^{\theta} d \theta_{1} \int_{0}^{\theta_{1}} d \theta_{2} \int_{0}^{\theta_{2}} d \theta_{3} \int_{0}^{\theta_{3}} d \theta_{4}
\end{aligned}
$$

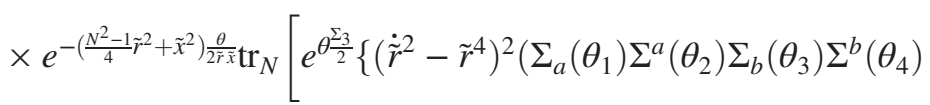

$$
\begin{aligned}
& \left.+\Sigma_{a}\left(\theta_{1}\right) \Sigma_{b}\left(\theta_{2}\right) \Sigma^{b}\left(\theta_{3}\right) \Sigma^{a}\left(\theta_{4}\right)+\Sigma_{a}\left(\theta_{1}\right) \Sigma_{b}\left(\theta_{2}\right) \Sigma^{a}\left(\theta_{3}\right) \Sigma^{b}\left(\theta_{4}\right)\right) \\
& \left.\left.+4 \dot{\tilde{r}}^{2} \tilde{r}^{4}\left(\Sigma_{a}\left(\theta_{1}\right) \Sigma^{a}\left(\theta_{2}\right) \Sigma_{b}\left(\theta_{3}\right) \Sigma^{b}\left(\theta_{4}\right)-\Sigma_{a}\left(\theta_{1}\right) \Sigma_{b}\left(\theta_{2}\right) \Sigma^{b}\left(\theta_{3}\right) \Sigma^{a}\left(\theta_{4}\right)\right)\right\}\right]
\end{aligned}
$$

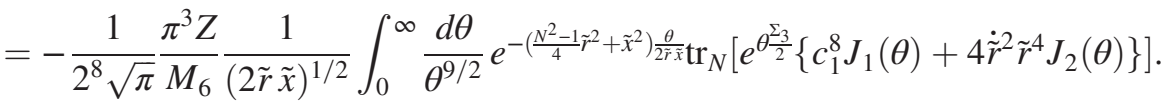

Here we used the energy conservation (13), which is written as $-\dot{\tilde{r}}^{2}+\tilde{r}^{4}=c_{1}^{4}$ for Euclidean time. Therefore, the coefficient of the $J_{1}(\theta)$-term in the trace is time independent. $J_{1}(\theta)$ and $J_{2}(\theta)$ are a power series of $\Sigma_{3}^{n}$ and their explicit forms are given in Appendix A. Now we take the large $N$ limit by keeping the size of the fuzzy sphere. Then the trace is transformed into an integral,

$\operatorname{tr}\left(e^{\theta \frac{\Sigma_{3}}{2}} \Sigma_{3}^{n}\right)=2^{n} \frac{d^{n}}{d \theta^{n}} \operatorname{tr}\left(e^{\theta^{\frac{\Sigma_{3}}{2}}}\right) \sim 2^{n+1} \frac{d^{n}}{d \theta^{n}}\left(\frac{\sinh \left(\frac{N \theta}{2}\right)}{\theta}\right)$.

Here the representation of $\Sigma_{3}$ was chosen as $\left(\Sigma_{3}\right)_{m, n}=$ $(N-2 m+1) \delta_{m, n}$. Finally, by inserting the above equation into Eq. (40), we obtain the effective potential of the time independent part at $M_{1}^{4}$, i.e., the $J_{1}$ part, as

$$
\begin{aligned}
\left.V_{\mathrm{eff}}\right|_{M^{4}, J_{1}} \sim & -\frac{\pi^{3} N Z}{2^{11} M_{6}} \frac{N^{4} c_{1}^{8}}{\left(2 \tilde{R}_{\mathrm{sph}} \tilde{x}\right)^{1 / 2}}\left(\sqrt{\frac{\tilde{R}_{\mathrm{sph}}}{2 \tilde{x}}+\frac{\tilde{x}}{2 \tilde{R}_{\mathrm{sph}}}+1}\right. \\
& \left.-\sqrt{\frac{\tilde{R}_{\mathrm{sph}}}{2 \tilde{x}}+\frac{\tilde{x}}{2 \tilde{R}_{\mathrm{sph}}}-1}\right),
\end{aligned}
$$

where $\tilde{R}_{\mathrm{sph}} \sim \tilde{r} N / 2$ is typical mass scale of the fuzzy sphere. The final expression is derived by employing 
Mathematica. Although $\tilde{R}_{\text {sph }}$ is time dependent, we assume that the fuzzy sphere is oscillating slowly around $t=0$ and $\tilde{R}_{\text {sph }}$ is finite. Then if we take $\tilde{R}_{\text {sph }} \ll \tilde{x}$, where the test D0-brane is far from the fuzzy sphere in three dimensions, the above effective potential becomes

$\left.V_{\mathrm{eff}}\right|_{M^{4}, J_{1}} \sim-\frac{\pi^{3} N Z}{2^{11} M_{6}} \frac{N^{4} c_{1}^{8}}{\tilde{x}} \sim-\frac{\pi^{3}(N Z)}{2^{5} M_{6}}\left(\frac{E_{\mathrm{sph}} \lambda}{(N Z)^{2}}\right)^{2} \frac{1}{\tilde{x}}$.

In the above, Eq. (22) is used. This result should be compared with that of the near horizon geometry of the smeared black 0-brane.

\section{B. Effective potential via smeared fuzzy cylinder}

In this subsection, we analyze the effective potential between the fuzzy cylinder and the test D0-brane. The $Q^{2}$ in Eq. (28) is evaluated as

$$
Q^{2}=\left(\tilde{\rho}^{2}+\tilde{x}^{2}\right) \mathbf{1}_{N}+\tilde{l}^{2} \Xi_{3}^{2}-2 \tilde{l} \tilde{x} \Xi_{3},
$$

and the field strength $G_{\alpha \beta}(\ell)$ is given by

$$
G_{\alpha \beta}(\ell)=\left(\begin{array}{ccc}
0 & \dot{\tilde{\rho}} \Xi_{b}(\ell) & 0 \\
-\dot{\tilde{\rho}} \Xi_{a}(\ell) & 0 & -\tilde{l} \tilde{\rho} \epsilon_{a c} \Xi^{c}(\ell) \\
0 & \tilde{l} \tilde{\rho} \epsilon_{b c} \Xi^{c}(\ell) & 0
\end{array}\right),
$$

where $a, b=1,2$ and $\epsilon_{a b}$ is an antisymmetric tensor. $\Xi_{a}(\ell) \equiv e^{\ell Q^{2}} \Xi_{a} e^{-\ell Q^{2}}$ is explicitly evaluated as

$$
\begin{aligned}
\left(\Xi_{1}(\ell)\right)_{m n} & =\frac{1}{2} e^{\ell \lambda_{m}} \delta_{m+1, n}+\frac{1}{2} e^{-\ell \lambda_{n}} \delta_{m, n+1}, \\
\left(\Xi_{2}(\ell)\right)_{m n} & =-\frac{i}{2} e^{\ell \lambda_{m}} \delta_{m+1, n}+\frac{i}{2} e^{-\ell \lambda_{n}} \delta_{m, n+1}, \\
\lambda_{m} & \equiv-2 \tilde{l}^{2}\left(m+\frac{\tilde{x}}{\tilde{l}}\right)-\tilde{l}^{2} .
\end{aligned}
$$

By inserting the above expressions into Eq. (35), the effective potential at $M^{4}$ order is evaluated as follows:

$$
\begin{aligned}
\left.V_{\mathrm{eff}}\right|_{M^{4}}= & -\frac{1}{2^{4} \sqrt{\pi}} \frac{\pi^{3} Z}{M_{6}} \int_{0}^{\infty} \frac{d \ell}{\ell^{9 / 2}} \int_{0}^{\ell} d \ell_{1} \int_{0}^{\ell_{1}} d \ell_{2} \int_{0}^{\ell_{2}} d \ell_{3} \int_{0}^{\ell_{3}} d \ell_{4} \\
& \times e^{-\ell\left(\tilde{\rho}^{2}+\tilde{x}^{2}\right)} \operatorname{tr}\left[e ^ { - \ell ( \tilde { l } ^ { 2 } \Xi _ { 3 } ^ { 2 } - 2 \tilde { l } \tilde { x } \Xi _ { 3 } ) } \left\{( \dot { \tilde { \rho } } ^ { 2 } - \tilde { l } ^ { 2 } \tilde { \rho } ^ { 2 } ) ^ { 2 } \left(\Xi_{a}\left(\ell_{1}\right) \Xi^{a}\left(\ell_{2}\right) \Xi_{b}\left(\ell_{3}\right) \Xi^{b}\left(\ell_{4}\right)\right.\right.\right. \\
& \left.+\Xi_{a}\left(\ell_{1}\right) \Xi_{b}\left(\ell_{2}\right) \Xi^{b}\left(\ell_{3}\right) \Xi^{a}\left(\ell_{4}\right)+\Xi_{a}\left(\ell_{1}\right) \Xi_{b}\left(\ell_{2}\right) \Xi^{a}\left(\ell_{3}\right) \Xi^{b}\left(\ell_{4}\right)\right) \\
& \left.\left.+4 \tilde{l}^{2} \tilde{\rho}^{2} \dot{\tilde{\rho}}^{2}\left(\Xi_{a}\left(\ell_{1}\right) \Xi^{a}\left(\ell_{2}\right) \Xi_{b}\left(\ell_{3}\right) \Xi^{b}\left(\ell_{4}\right)-\Xi_{a}\left(\ell_{1}\right) \Xi_{b}\left(\ell_{2}\right) \Xi^{b}\left(\ell_{3}\right) \Xi^{a}\left(\ell_{4}\right)\right)\right\}\right] \\
= & -\frac{1}{2^{4} \sqrt{\pi}} \frac{\pi^{3} Z}{M_{6}} \int_{0}^{\infty} \frac{d \ell}{\ell^{9 / 2}} e^{-\ell \tilde{\rho}^{2}} \operatorname{tr}_{N}\left[e^{-\ell \tilde{l}^{2}\left(\Xi_{3}-\frac{\tilde{l}}{\tilde{l}}\right)^{2}}\left\{\tilde{l}^{4} c_{3}^{4} L_{1}(\ell)+4 \tilde{l}^{2} \tilde{\rho}^{2} \dot{\tilde{\rho}}^{2} L_{2}(\ell)\right\}\right] .
\end{aligned}
$$

Here we used the energy conservation (19) for the Euclidean time, that is, $-\dot{\tilde{\rho}}^{2}+\tilde{l}^{2} \tilde{\rho}^{2}=\tilde{l}^{2} c_{3}^{2}$. Because of this relation, the $L_{1}(\ell)$ part in the trace is time independent. $L_{1}(\ell)$ and $L_{2}(\ell)$ are diagonal matrices and their explicit forms are given in Appendix B. Now we take the density of D0-branes per length infinite. Then the trace is transformed into the integral,

$$
\operatorname{tr}\left(e^{-\ell \tilde{l}^{2}\left(\Xi_{3}-\frac{\tilde{I}}{l} 1\right)^{2}} F\right) \sim \int_{-\infty}^{\infty} d \zeta e^{-\ell \tilde{l}^{2} \zeta^{2}} f\left(-2 \tilde{l}^{2} \zeta-\tilde{l}^{2}\right) .
$$

Here $F$ is some diagonal matrix whose component is given by $F_{m n}=f\left(\lambda_{m}\right) \delta_{m, n}$. Since the length of the fuzzy cylinder is infinite, the range of the integral also becomes infinite. Then the $\tilde{x}$ dependence disappears by shifting the origin. Finally, by inserting the above equation into Eq. (47), the time independent part of the effective potential at the $M_{1}^{4}$ order becomes

$\left.V_{\mathrm{eff}}\right|_{M^{4}, L_{1}} \sim-\frac{\pi^{3} Z}{2^{4} M_{6}} \tilde{l}^{4} c_{3}^{4} \int_{-\infty}^{\infty} d \zeta\left(\frac{1}{8 \tilde{l}|\zeta|}+\mathcal{O}\left(\zeta^{-2}\right)\right)$.
The last expression is derived by employing Mathematica. The function in the bracket depends on $\tilde{\rho} / \tilde{l}$ and is expanded around $\zeta=\infty$. The explicit form of the function is complicated, but it is regular around the origin. The effective potential of Eq. (49) shows that there is no force between the fuzzy cylinder and the test D0-brane at leading order.

\section{COMPARISON WITH THE GRAVITY SIDE}

In this section we review the properties of the smeared black 0-brane and compare the effective potentials for the test D0-brane with those of the previous section. The black 0-brane solution is obtained by boosting the 11 dimensional black hole along the 11th direction. In a similar way, the smeared black 0-brane solution can be constructed by boosting the smeared black hole along the 11th direction [31]. The metric, the dilaton field, and the $\mathrm{R}-\mathrm{R}$ one-form field for the smeared black 0-brane are written as 


$$
\begin{aligned}
d s_{10}^{2} & =-H^{-\frac{1}{2}} F d t^{2}+H^{\frac{1}{2}}\left(F^{-1} d r^{2}+r^{2} d \Omega_{2}^{2}+d x_{u}^{2}\right), \\
e^{\phi} & =H^{\frac{3}{4}}, \quad C^{(1)}=\sqrt{1+\alpha}\left(1-H^{-1}\right) d t, \\
H & =1+\frac{r_{-}}{r}, \quad F=1-\frac{r_{-} \alpha}{r} .
\end{aligned}
$$

Here $x_{u}(u=4, \ldots, 9)$ labels the smeared directions. The solution has two parameters $r_{-}$and $\alpha$, and the latter corresponds to the boost parameter.

Let us evaluate physical quantities of the smeared black 0 -brane. The event horizon is located at $r_{\mathrm{h}}=r_{-} \alpha$, and the temperature $T$ and electric potential $\Phi$ are given by

$$
\begin{aligned}
& T=\left.\frac{1}{4 \pi} H^{-1 / 2} \frac{d F}{d r}\right|_{r_{\mathrm{h}}}=\frac{1}{4 \pi r_{-} \alpha} \sqrt{\frac{\alpha}{1+\alpha}}, \\
& \Phi=\left.C_{t}^{(1)}\right|_{r_{\mathrm{h}}}=\frac{1}{\sqrt{1+\alpha}} .
\end{aligned}
$$

The Arnowitt-Deser-Misner (ADM) mass $M$ and the R-R charge $Q$ of the smeared black 0 -brane are evaluated as usual, and the results become

$M=\frac{4 \pi V_{6}}{2 \kappa_{10}^{2}} r_{-} \alpha\left(2+\frac{1}{\alpha}\right), \quad Q=\frac{4 \pi V_{6}}{2 \kappa_{10}^{2}}(\sqrt{1+\alpha}) r_{-}$.

$V_{6}=\prod_{u=4}^{9} 2 \pi R_{u}$ is the volume of the compactified six directions and $2 \kappa_{10}^{2}=(2 \pi)^{7} \ell_{s}^{8} g_{s}^{2}$ is the 10 dimensional gravitational constant. $\ell_{s}$ is the string length and $g_{s}$ is the string coupling constant. The extremal limit corresponds to $\alpha \rightarrow 0$.

Next let us consider the near horizon limit of the smeared black 0-brane. The near horizon limit is defined so that physical quantities of the dual gauge theory become finite [19]. Thus, the near horizon limit for the black 0-brane is defined as [22]

$r \rightarrow 0 \quad$ with $\quad U=\frac{r}{\ell_{s}^{2}} \quad$ and $\quad \lambda=\frac{g_{s} N^{\prime}}{(2 \pi)^{2} \ell_{s}^{3}}$ fixed.

Here $U$ is a typical energy scale of the system. The 't Hooft coupling is denoted by $\lambda=g_{\mathrm{YM}}^{2} N^{\prime}$ and $N^{\prime}=N Z$ is the number of the smeared D0-branes. Note that the energy scale at the horizon $U_{\mathrm{h}}=\frac{r_{-} \alpha}{\ell_{s}^{2}}$ is also fixed. In terms of $\alpha$ and $r_{-}$, the near horizon limit is defined as

$$
\alpha \rightarrow 0 \text { with } \frac{r}{r_{-} \alpha} \text { and } \frac{r_{-} \alpha}{\ell_{s}^{2}} \text { fixed. }
$$

Let us examine the $\alpha \rightarrow 0$ limit more carefully. Since the black 0-brane corresponds to the D0-brane, the R-R charge of the D0-branes should be

$$
Q=\frac{N^{\prime}}{\ell_{s} g_{s}} .
$$

Furthermore, since the black 0-brane is smeared into six spatial directions, we should fix the typical mass scale for the compactified six spatial directions. Namely, we fix $M_{6}=\prod_{u=4}^{9} 2 \pi \tilde{R}_{u}$. Then, in the near horizon limit, $\alpha$ goes to zero like

$$
\alpha \rightarrow \frac{M_{6} U_{\mathrm{h}}}{2 \pi^{2} \lambda} \ell_{s}^{4} .
$$

Note that $r_{-}$goes to the infinity through the relation $r_{-}=U_{\mathrm{h}} \ell_{s}^{2} / \alpha$, and $H$ and $F$ in Eq. (50) are written as

$$
H \rightarrow \frac{1}{\alpha} \frac{U_{\mathrm{h}}}{U}, \quad F=1-\frac{U_{\mathrm{h}}}{U} .
$$

The thermodynamics of the near horizon geometry of the smeared black 0-brane becomes as follows. The temperature in (51) becomes

$$
T=\frac{M_{6}^{1 / 2}}{4 \sqrt{2} \pi^{2} \lambda^{1 / 2} U_{\mathrm{h}}^{1 / 2}},
$$

and the internal energy $E=M-Q$ is expressed as

$$
\frac{E}{N^{\prime 2}}=\frac{3 M_{6} U_{\mathrm{h}}}{16 \pi^{4} \lambda^{2}}=\frac{3 M_{6}^{2}}{2(2 \pi)^{8} \lambda^{3} T^{2}} .
$$

Finally, we examine a test D0-brane moving around the smeared black 0-brane. Let us consider the potential energy for the test D0-brane, which is moving only along the radial direction. With this assumption, the Lagrangian for the D0-brane in the background of the smeared black 0-brane (50) becomes

$$
\begin{aligned}
\mathcal{L} & =-T_{0} e^{-\phi} \sqrt{-g_{\mu \nu} \dot{x}^{\mu} \dot{x}^{\nu}}-T_{0} C_{t}^{(1)} \\
& =-T_{0} e^{-\phi} H^{-\frac{1}{4}} F^{\frac{1}{2}} \sqrt{1-H F^{-2} \dot{r}^{2}}-T_{0} \sqrt{1+\alpha}\left(1-H^{-1}\right) .
\end{aligned}
$$

And the momentum conjugate to $r$ is defined as

$$
p_{r}=\frac{\partial \mathcal{L}}{\partial \dot{r}}=T_{0} H^{-1} F^{\frac{1}{2}} \frac{H F^{-2} \dot{r}}{\sqrt{1-H F^{-2} \dot{r}^{2}}} .
$$

By using the above equation, $\dot{r}$ is expressed in terms of $p_{r}$, and the Hamiltonian of the D0-brane is evaluated as

$\mathcal{H}=H^{-1} F^{\frac{1}{2}} \sqrt{T_{0}^{2}+H F p_{r}^{2}}+T_{0} \sqrt{1+\alpha}\left(1-H^{-1}\right)$.

If the momentum is small enough, we can expand the above with respect to the momentum and read off the potential energy as

$$
V=T_{0} H^{-1} F^{\frac{1}{2}}+T_{0} \sqrt{1+\alpha}\left(1-H^{-1}\right) .
$$

The first term corresponds to the attractive force by the gravity and the second term corresponds to the repulsive force due to the R-R background. In the classical (or $1 \ll r$ ) and near horizon limits, the potential becomes 


$$
\begin{aligned}
V-T_{0} & \sim T_{0} \alpha \frac{U}{U_{\mathrm{h}}}(\sqrt{F}-1)+T_{0} \frac{\alpha}{2} \sim-\frac{M_{6} U_{\mathrm{h}}^{2} N^{\prime}}{64 \pi^{4} \lambda^{2}} \frac{1}{U} \\
& =-\frac{4 \pi^{4}(N Z)}{M_{6}}\left(\frac{E \lambda}{3(N Z)^{2}}\right)^{2} \frac{1}{U} \\
& =-\frac{2 \pi^{3}(N Z)}{9 M_{6}}\left(\frac{E \lambda}{(N Z)^{2}}\right)^{2} \frac{1}{\tilde{x}} .
\end{aligned}
$$

The rest mass of the D0-brane is subtracted in the above, since it is divergent constant in the near horizon limit. In the last line, we used Eq. (59), $N^{\prime}=N Z$ and $U=x / \ell_{s}^{2}=2 \pi \tilde{x}$. The qualitative feature of Eq. (64) surely matches with Eq. (43).

So far we have smeared the four dimensional black hole along six spatial directions, and boosted it along the 11th direction. And the solution is given by Eq. (50). Then we might try to smear the three dimensional black hole along seven spatial directions, and boost it along the 11th direction. However, there is no three dimensional black hole which is asymptotic to the flat spacetime [37]. This means that there is no black 0-brane which is smeared along seven spatial directions. So the effective potential between the black 0 -brane and test D0-brane should be trivial. This is consistent with the result (49), which does not depend on $\tilde{x}$.

\section{CONCLUSION AND DISCUSSION}

In this paper, we conjectured that the fuzzy configurations of D0-branes in the BFSS matrix model would correspond to the microstates of the smeared black 0-brane in the near horizon limit. The fuzzy configurations are constructed by smearing the fuzzy objects in three dimensions into six spatial directions. Since the fuzzy objects have the internal energy compared with the static case, they are time dependent and non-BPS states. Thus, the fuzzy configurations would correspond to the microstates of the nonextremal black 0-brane in the near horizon limit. As a nontrivial check, we evaluated the one-loop effective potential for the test D0-brane in the background of the smeared fuzzy sphere. We found that the effective potential for the test D0-brane behaves like Eq. (43) in the BFSS matrix model. On the other hand, the effective potential was also evaluated from the gravity side like Eq. (64). These two results match up to the numerical factor, so this shows evidence that the smeared fuzzy objects are the microstates of the black hole. Furthermore, we also evaluated the one-loop effective potential for the test D0-brane in the background of the smeared fuzzy cylinder. In this case, the effective potential becomes trivial, and it agrees with the fact that there is no asymptotically flat black hole in three dimensions. Thus, the conjecture is supported at least by one-loop calculation of the effective potential for the test D0-brane, but following checks below will be necessary for further confirmation.

Although the qualitative features of the smeared fuzzy objects match with those of the smeared black 0-brane in the gravity side, we still have the discrepancy in the numerical coefficients. This is similar to the case of nonextremal black 3-brane thermodynamics [38]. In order to cure this problem from the gravity side, we need to take into account $\alpha^{\prime}$ corrections in type IIA superstring theory. This will modify the form of $F(r)$ in the metric (50) and the mass of the black 0-brane will be renormalized as argued in Ref. [39].

Notice that, if we take into account $\alpha^{\prime}$ and $g_{s}$ corrections, $F(r)$ and $H(r)$ should also be corrected by terms like $r^{-n}(n=2,3, \ldots)$. Those terms become important for the small $r$ region $\left(r \sim r_{\mathrm{h}}\right)$, and should be compared with the effective potential with higher loop corrections in the BFSS matrix model. However, it is very hard to check this statement so far because we need precise knowledge of $\alpha^{\prime}$ and $g_{s}$ corrections in type IIA superstring theory and higher loop calculations in the BFSS matrix model in the background of the fuzzy sphere.

In this paper, we focused on irreducible representations in Eq. (3). It is possible, however, to consider reducible ones which correspond to multiple fuzzy objects. For example, we divide the size of the matrix $N$ for the fuzzy object into $n$ pieces like $N=\sum_{i=1}^{n} N_{i}$, and prepare parameters $d_{i}(i=1, \ldots, n)$ so as to satisfy $N^{3} c_{1}^{4}=\sum_{i=1}^{n} N_{i}^{3} d_{i}^{4}$. Then we construct the fuzzy object out of $n$ fuzzy spheres, each of which has the matrix size $N_{i}$ and the internal energy $E_{\mathrm{sph},} i=N_{i}^{3} d_{i}^{4} /\left(8 g_{\mathrm{YM}}^{2}\right)$. This fuzzy object has the same internal energy as Eq. (13) in the large $N_{i}$ limit. And the effective potential for the test D0-brane (43) is modified as follows:

$$
\left.V_{\mathrm{eff}}\right|_{M^{4}, J_{1}} \sim-\frac{\pi^{3} Z}{2^{11} M_{6}} \frac{\sum_{i=1}^{n} N_{i}^{5} d_{i}^{8}}{\tilde{x}} .
$$

The numerical coefficient is different from Eq. (43), but the order is almost the same. For instance, if we choose $N_{i} / N \sim$ $1 / n$ and $\left(N_{i} / N\right)^{3}\left(d_{i} / c_{1}\right)^{4} \sim 1 / n$ for all $i$, we obtain $\sum_{i=1}^{n}\left(N_{i} / N\right)^{5}\left(d_{i} / c_{1}\right)^{8} \sim 1$. Since these configurations give the same internal energy as the single fuzzy sphere, these will be the microstates of the smeared black 0-brane in the near horizon limit. In practice, it is very difficult to count the number of microstates since these multiple fuzzy spheres are dynamical and interacting with each other. ${ }^{2}$ Note that this proposal is similar to the notion of the fuzz ball for the black hole [41].

In this paper, we considered the fuzzy objects which have axial symmetry. It is possible to relax this ansatz to construct a generic configuration [42], and it will also contribute to the microstates of the black hole. For future directions, it is interesting to examine the multishell model which is proposed as an alternative black hole evaporation mechanism in Refs. $[43,44]$. The similar situation can be analyzed by using the multiple fuzzy spheres discussed in the above. Notice that the time evolutions of the fuzzy configurations are quite complicated even for the two D0-branes case (See Fig. 1). This shows that the black hole has a chaotic behavior as recently studied in Refs. [45-48].

\footnotetext{
${ }^{2}$ For the static case, the von Neumann entropy of multiple fuzzy spheres in $S^{3}$ is evaluated in Ref. [40].
} 


\section{ACKNOWLEDGMENTS}

The author would like to thank National Taiwan University (NTU), National Center for Theoretical Sciences (NCTS), and Dublin Institute for Advanced Studies (DIAS) for their warm hospitality. Especially the author would like to thank Yuhma Asano, Masanori Hanada, Pei-Ming Ho, Denjoe O'Connor, and Tadashi
Okazaki. This work was partially supported by Japan Society for the Promotion of Science, Grant-in-Aid for Scientific Research (C) Grant No. JP17K05405.

\section{APPENDIX A: CALCULATION OF $\boldsymbol{J}_{\mathbf{1}}$ AND $\boldsymbol{J}_{\mathbf{2}}$}

The definition of $J_{1}(\theta)$ and explicit expression are given as follows:

$$
\begin{aligned}
J_{1}(\theta) \equiv & \int_{0}^{\theta} d \theta_{1} \int_{0}^{\theta_{1}} d \theta_{2} \int_{0}^{\theta_{2}} d \theta_{3} \int_{0}^{\theta_{3}} d \theta_{4}\left(\Sigma_{a}\left(\theta_{1}\right) \Sigma^{a}\left(\theta_{2}\right) \Sigma_{b}\left(\theta_{3}\right) \Sigma^{b}\left(\theta_{4}\right)\right. \\
& \left.+\Sigma_{a}\left(\theta_{1}\right) \Sigma_{b}\left(\theta_{2}\right) \Sigma^{b}\left(\theta_{3}\right) \Sigma^{a}\left(\theta_{4}\right)+\Sigma_{a}\left(\theta_{1}\right) \Sigma_{b}\left(\theta_{2}\right) \Sigma^{a}\left(\theta_{3}\right) \Sigma^{b}\left(\theta_{4}\right)\right) \\
= & \int_{0}^{\theta} d \theta_{1} \int_{0}^{\theta_{1}} d \theta_{2} \int_{0}^{\theta_{2}} d \theta_{3} \int_{0}^{\theta_{3}} d \theta_{4}\left(-4 \sinh \left(\theta_{1}-\theta_{2}+\theta_{3}-\theta_{4}\right)\left(\left(N^{2}-1\right) \Sigma_{3}-\Sigma_{3}^{3}\right)\right. \\
& -8 \sinh \left(\theta_{1}+\theta_{2}-\theta_{3}-\theta_{4}\right)\left(\left(N^{2}-3\right) \Sigma_{3}-\Sigma_{3}^{3}\right)+\cosh \left(\theta_{1}+\theta_{2}-\theta_{3}-\theta_{4}\right)\left(\left(N^{2}-9\right)\left(N^{2}-1\right)-2\left(N^{2}-11\right) \Sigma_{3}^{2}+\Sigma_{3}^{4}\right) \\
& +\cosh \left(\theta_{1}-\theta_{2}+\theta_{3}-\theta_{4}\right)\left(\left(N^{2}-1\right)^{2}-2\left(N^{2}-3\right) \Sigma_{3}^{2}+\Sigma_{3}^{4}\right)+\cosh \left(\theta_{1}-\theta_{2}-\theta_{3}+\theta_{4}\right)\left(\left(N^{2}-1\right)^{2}-2\left(N^{2}+1\right) \Sigma_{3}^{2}+\Sigma_{3}^{4}\right) \\
& -2 \sinh \left(\theta_{1}-\theta_{2}\right) \Sigma_{3}^{3}+2 \sinh \left(\theta_{1}-\theta_{3}\right)\left(\left(N^{2}-1\right) \Sigma_{3}-2 \Sigma_{3}^{3}\right)+2 \sinh \left(\theta_{1}-\theta_{4}\right)\left(2\left(N^{2}-3\right) \Sigma_{3}-3 \Sigma_{3}^{3}\right)-2 \sinh \left(\theta_{2}-\theta_{3}\right) \Sigma_{3}^{3} \\
& +2 \sinh \left(\theta_{2}-\theta_{4}\right)\left(\left(N^{2}-1\right) \Sigma_{3}-2 \Sigma_{3}^{3}\right)-2 \sinh \left(\theta_{3}-\theta_{4}\right) \Sigma_{3}^{3}+\cosh \left(\theta_{1}-\theta_{2}\right)\left(\left(N^{2}-1\right) \Sigma_{3}^{2}-\Sigma_{3}^{4}\right) \\
& +\cosh \left(\theta_{1}-\theta_{3}\right)\left(\left(N^{2}-5\right) \Sigma_{3}^{2}-\Sigma_{3}^{4}\right)+\cosh \left(\theta_{1}-\theta_{4}\right)\left(4\left(N^{2}-1\right)+\left(N^{2}-13\right) \Sigma_{3}^{2}-\Sigma_{3}^{4}\right)+\cosh \left(\theta_{2}-\theta_{3}\right)\left(\left(N^{2}-1\right) \Sigma_{3}^{2}-\Sigma_{3}^{4}\right) \\
& \left.+\cosh \left(\theta_{2}-\theta_{4}\right)\left(\left(N^{2}-5\right) \Sigma_{3}^{2}-\Sigma_{3}^{4}\right)+\cosh \left(\theta_{3}-\theta_{4}\right)\left(\left(N^{2}-1\right) \Sigma_{3}^{2}-\Sigma_{3}^{4}\right)+3 \Sigma_{3}^{4}\right) \\
= & \left(N^{2}-1\right)\left(\frac{N^{2}-9}{4} \cosh (2 \theta)+2 \theta^{2} \cosh \theta-\left(N^{2}-5\right) \cosh \theta+\frac{3 N^{2}-11}{4}\right) \mathbf{1}_{N}-2\left(N^{2}-3\right)\left(\sinh (2 \theta)-\theta^{2} \sinh \theta-2 \sinh \theta\right) \Sigma_{3} \\
& -\left(\frac{N^{2}-11}{2} \cosh (2 \theta)-\frac{N^{2}-13}{2} \theta^{2} \cosh \theta-2\left(N^{2}-5\right) \cosh \theta+\frac{N^{2}-1}{2} \theta^{2}+\frac{3\left(N^{2}-3\right)}{2}\right) \Sigma_{3}^{2} \\
& +\left(2 \sinh (2 \theta)-3 \theta^{2} \sinh \theta-4 \sinh \theta+\theta^{3}\right) \Sigma_{3}^{3}+\left(\frac{1}{4} \cosh (2 \theta)-\frac{1}{2} \theta^{2} \cosh \theta-\cosh \theta+\frac{1}{8} \theta^{4}+\frac{1}{2} \theta^{2}+\frac{3}{4}\right) \Sigma_{3}^{4} .
\end{aligned}
$$

The definition of $J_{2}(\theta)$ and explicit expression are given as follows:

$$
\begin{aligned}
J_{2}(\theta) \equiv & \int_{0}^{\theta} d \theta_{1} \int_{0}^{\theta_{1}} d \theta_{2} \int_{0}^{\theta_{2}} d \theta_{3} \int_{0}^{\theta_{3}} d \theta_{4}\left(\Sigma_{a}\left(\theta_{1}\right) \Sigma^{a}\left(\theta_{2}\right) \Sigma_{b}\left(\theta_{3}\right) \Sigma^{b}\left(\theta_{4}\right)-\Sigma_{a}\left(\theta_{1}\right) \Sigma_{b}\left(\theta_{2}\right) \Sigma^{b}\left(\theta_{3}\right) \Sigma^{a}\left(\theta_{4}\right)\right) \\
= & \int_{0}^{\theta} d \theta_{1} \int_{0}^{\theta_{1}} d \theta_{2} \int_{0}^{\theta_{2}} d \theta_{3} \int_{0}^{\theta_{3}} d \theta_{4}\left(4 \sinh \left(\theta_{1}+\theta_{2}-\theta_{3}-\theta_{4}\right)\left(\left(N^{2}-3\right) \Sigma_{3}-\Sigma_{3}^{3}\right)\right. \\
& -\frac{1}{2} \cosh \left(\theta_{1}+\theta_{2}-\theta_{3}-\theta_{4}\right)\left(\left(N^{2}-9\right)\left(N^{2}-1\right)-2\left(N^{2}-11\right) \Sigma_{3}^{2}+\Sigma_{3}^{4}\right)+\frac{1}{2} \cosh \left(\theta_{1}-\theta_{2}-\theta_{3}+\theta_{4}\right)\left(\left(N^{2}-1\right)^{2}\right. \\
& \left.-2\left(N^{2}+1\right) \Sigma_{3}^{2}+\Sigma_{3}^{4}\right)-2 \sinh \left(\theta_{1}-\theta_{2}\right) \Sigma_{3}^{3}-2 \sinh \left(\theta_{1}-\theta_{4}\right)\left(2\left(N^{2}-3\right) \Sigma_{3}-3 \Sigma_{3}^{3}\right)+2 \sinh \left(\theta_{2}-\theta_{3}\right) \Sigma_{3}^{3} \\
& -2 \sinh \left(\theta_{3}-\theta_{4}\right) \Sigma_{3}^{3}+\cosh \left(\theta_{1}-\theta_{2}\right)\left(\left(N^{2}-1\right) \Sigma_{3}^{2}-\Sigma_{3}^{4}\right)-\cosh \left(\theta_{1}-\theta_{4}\right)\left(4\left(N^{2}-1\right)+\left(N^{2}-13\right) \Sigma_{3}^{2}-\Sigma_{3}^{4}\right) \\
& \left.-\cosh \left(\theta_{2}-\theta_{3}\right)\left(\left(N^{2}-1\right) \Sigma_{3}^{2}-\Sigma_{3}^{4}\right)+\cosh \left(\theta_{3}-\theta_{4}\right)\left(\left(N^{2}-1\right) \Sigma_{3}^{2}-\Sigma_{3}^{4}\right)\right) \\
= & \left(N^{2}-1\right)\left(-\frac{N^{2}-9}{8} \cosh (2 \theta)-2 \theta^{2} \cosh \theta+\frac{N^{2}+7}{2} \theta \sinh \theta-8 \cosh \theta-\frac{N^{2}-1}{4} \theta^{2}+\frac{N^{2}+55}{8}\right) \mathbf{1}_{N} \\
& +\left(N^{2}-3\right)\left(\sinh (2 \theta)-2 \theta^{2} \sinh \theta+4 \theta \cosh \theta-8 \sinh \theta+2 \theta\right) \Sigma_{3}+\left(\frac{N^{2}-11}{4} \cosh (2 \theta)-\frac{N^{2}-13}{2} \theta^{2} \cosh \theta\right. \\
& \left.-2\left(N^{2}-13\right) \cosh \theta+\left(N^{2}-15\right) \theta \sinh \theta+\theta^{2}+\frac{7 N^{2}-93}{4}\right) \Sigma_{3}^{2}+\left(-\sinh (2 \theta)+3 \theta^{2} \sinh \theta-8 \theta \cosh \theta\right. \\
& \left.+12 \sinh \theta+\frac{1}{3} \theta^{3}-2 \theta\right) \Sigma_{3}^{3}+\left(-\frac{1}{8} \cosh (2 \theta)+\frac{1}{2} \theta^{2} \cosh \theta-\frac{3}{2} \theta \sinh \theta+2 \cosh \theta+\frac{1}{4} \theta^{2}-\frac{15}{8}\right) \Sigma_{3}^{4} .
\end{aligned}
$$




\section{APPENDIX B: CALCULATION OF $L_{1}$ AND $L_{2}$}

The definition of $L_{1}(\ell)$ and explicit expression are given as follows:

$$
\begin{aligned}
& \left(L_{1}(\ell)\right)_{m n} \equiv \int_{0}^{\ell} d \ell_{1} \int_{0}^{\ell_{1}} d \ell_{2} \int_{0}^{\ell_{2}} d \ell_{3} \int_{0}^{\ell_{3}} d \ell_{4}\left(\Xi_{a}\left(\ell_{1}\right) \Xi^{a}\left(\ell_{2}\right) \Xi_{b}\left(\ell_{3}\right) \Xi^{b}\left(\ell_{4}\right)\right. \\
& \left.+\Xi_{a}\left(\ell_{1}\right) \Xi_{b}\left(\ell_{2}\right) \Xi^{b}\left(\ell_{3}\right) \Xi^{a}\left(\ell_{4}\right)+\Xi_{a}\left(\ell_{1}\right) \Xi_{b}\left(\ell_{2}\right) \Xi^{a}\left(\ell_{3}\right) \Xi^{b}\left(\ell_{4}\right)\right)_{m n} \\
& =\frac{1}{2} \int_{0}^{\ell} d \ell_{1} \int_{0}^{\ell_{1}} d \ell_{2} \int_{0}^{\ell_{2}} d \ell_{3} \int_{0}^{\ell_{3}} d \ell_{4}\left(e^{\left(\ell_{1}-\ell_{2}+\ell_{3}-\ell_{4}\right) \lambda_{m}}+e^{\left(-\ell_{1}+\ell_{2}-\ell_{3}+\ell_{4}\right) \lambda_{m-1}}\right. \\
& \left.+e^{\left(\ell_{1}-\ell_{4}\right) \lambda_{m}+\left(\ell_{2}-\ell_{3}\right) \lambda_{m+1}}+e^{\left(-\ell_{1}+\ell_{2}\right) \lambda_{m-1}+\left(\ell_{3}-\ell_{4}\right) \lambda_{m}}+e^{\left(\ell_{1}-\ell_{2}\right) \lambda_{m}-\left(\ell_{3}-\ell_{4}\right) \lambda_{m-1}}+e^{\left(-\ell_{1}+\ell_{4}\right) \lambda_{m-1}-\left(\ell_{2}-\ell_{3}\right) \lambda_{m-2}}\right) \delta_{m n} \\
& =\left\{\frac{\left(\lambda_{m-1}-\lambda_{m}\right)^{2}}{4 \lambda_{m-1}^{2} \lambda_{m}^{2}} \ell^{2}-\frac{\lambda_{m-2}-\lambda_{m-1}}{2 \lambda_{m-2} \lambda_{m-1}^{3}} \ell e^{-\ell \lambda_{m-1}}-\frac{\lambda_{m}-\lambda_{m+1}}{2 \lambda_{m}^{3} \lambda_{m+1}} \ell e^{\ell \lambda_{m}}\right. \\
& +\left(\frac{\left(\lambda_{m-1}-\lambda_{m}\right)\left(\lambda_{m-1}^{2}+\lambda_{m}^{2}\right)}{\lambda_{m-1}^{3} \lambda_{m}^{3}}+\frac{1}{2 \lambda_{m-1}^{2}\left(\lambda_{m-2}+\lambda_{m-1}\right)}-\frac{1}{2 \lambda_{m}^{2}\left(\lambda_{m}+\lambda_{m+1}\right)}\right) \ell \\
& +\left(\frac{1}{\lambda_{m-1}^{3}\left(\lambda_{m-1}+\lambda_{m}\right)}-\frac{3 \lambda_{m-2}^{2}-2 \lambda_{m-1} \lambda_{m-2}+\lambda_{m-1}^{2}}{2 \lambda_{m-2}^{2} \lambda_{m-1}^{4}}\right) e^{-\ell \lambda_{m-1}} \\
& -\left(\frac{3 \lambda_{m-1}+\lambda_{m}}{2 \lambda_{m}^{4}\left(\lambda_{m-1}+\lambda_{m}\right)}+\frac{\lambda_{m}-2 \lambda_{m+1}}{2 \lambda_{m}^{3} \lambda_{m+1}^{2}}\right) e^{\ell \lambda_{m}}+\frac{e^{-\ell\left(\lambda_{m-2}+\lambda_{m-1}\right)}}{2 \lambda_{m-2}^{2}\left(\lambda_{m-2}+\lambda_{m-1}\right)^{2}}+\frac{e^{\ell\left(\lambda_{m}+\lambda_{m+1}\right)}}{2 \lambda_{m+1}^{2}\left(\lambda_{m}+\lambda_{m+1}\right)^{2}} \\
& \left.+\left(\frac{\lambda_{m-2}\left(3 \lambda_{m-2}+4 \lambda_{m-1}\right)}{2 \lambda_{m-1}^{4}\left(\lambda_{m-2}+\lambda_{m-1}\right)^{2}}+\frac{3 \lambda_{m-1}^{3}-2 \lambda_{m} \lambda_{m-1}^{2}+2 \lambda_{m}^{2} \lambda_{m-1}-2 \lambda_{m}^{3}}{2 \lambda_{m-1}^{3} \lambda_{m}^{4}}-\frac{3 \lambda_{m}+2 \lambda_{m+1}}{2 \lambda_{m}^{3}\left(\lambda_{m}+\lambda_{m+1}\right)^{2}}\right)\right\} \delta_{m n} .
\end{aligned}
$$

The definition of $L_{2}(\ell)$ and explicit expression are given as follows.

$$
\begin{aligned}
\left(L_{2}(\ell)\right)_{m n} & \equiv \int_{0}^{\ell} d \ell_{1} \int_{0}^{\ell_{1}} d \ell_{2} \int_{0}^{\ell_{2}} d \ell_{3} \int_{0}^{\ell_{3}} d \ell_{4}\left(\Xi_{a}\left(\ell_{1}\right) \Xi^{a}\left(\ell_{2}\right) \Xi_{b}\left(\ell_{3}\right) \Xi^{b}\left(\ell_{4}\right)-\Xi_{a}\left(\ell_{1}\right) \Xi_{b}\left(\ell_{2}\right) \Xi^{b}\left(\ell_{3}\right) \Xi^{a}\left(\ell_{4}\right)\right)_{m n} \\
& =\left\{-\frac{1}{4 \lambda_{m-1} \lambda_{m}} \ell^{2}-\frac{\lambda_{m-1}-\lambda_{m}}{2 \lambda_{m-1}^{2} \lambda_{m}^{2}} \ell+\frac{1}{2 \lambda_{m-1}^{3}\left(\lambda_{m-1}+\lambda_{m}\right)} e^{-\ell \lambda_{m-1}}+\frac{1}{2 \lambda_{m}^{3}\left(\lambda_{m-1}+\lambda_{m}\right)} e^{\ell \lambda_{m}}-\frac{\lambda_{m-1}^{2}-\lambda_{m} \lambda_{m-1}+\lambda_{m}^{2}}{2 \lambda_{m-1}^{3} \lambda_{m}^{3}}\right\} \delta_{m n} .
\end{aligned}
$$

[1] J. Polchinski, D. Branes, and R.-R. Charges, Dirichlet Branes and Ramond-Ramond Charges, Phys. Rev. Lett. 75, 4724 (1995).

[2] A. Strominger and C. Vafa, Microscopic origin of the Bekenstein-Hawking entropy, Phys. Lett. B 379, 99 (1996).

[3] T. Banks, W. Fischler, S. H. Shenker, and L. Susskind, M theory as a matrix model: A conjecture, Phys. Rev. D 55, 5112 (1997).

[4] N. Ishibashi, H. Kawai, Y. Kitazawa, and A. Tsuchiya, A Large N reduced model as superstring, Nucl. Phys. B498, 467 (1997).

[5] R. Dijkgraaf, E. P. Verlinde, and H. L. Verlinde, Matrix string theory, Nucl. Phys. B500, 43 (1997).

[6] H. Itoyama and A. Tokura, USp(2k) matrix model: Nonperturbative approach to orientifolds, Phys. Rev. D 58, 026002 (1998).
[7] B. de Wit, J. Hoppe, and H. Nicolai, On quantum mechanics of supermembranes, Nucl. Phys. B305, 545 (1988).

[8] M. R. Douglas, D. N. Kabat, P. Pouliot, and S. H. Shenker, D-branes and short distances in string theory, Nucl. Phys. B485, 85 (1997).

[9] K. Becker and M. Becker, A Two loop test of M(atrix) theory, Nucl. Phys. B506, 48 (1997).

[10] K. Becker, M. Becker, J. Polchinski, and A. A. Tseytlin, Higher order graviton scattering in M(atrix) theory, Phys. Rev. D 56, R3174 (1997).

[11] L. Susskind, Another conjecture about M(atrix) theory, arXiv:hep-th/9704080.

[12] W. Taylor, M(atrix) theory: Matrix quantum mechanics as a fundamental theory, Rev. Mod. Phys. 73, 419 (2001). 
[13] T. Banks, W. Fischler, I. R. Klebanov, and L. Susskind, Schwarzschild Black Holes from Matrix Theory, Phys. Rev. Lett. 80, 226 (1998).

[14] T. Banks, W. Fischler, I. R. Klebanov, and L. Susskind, Schwarzschild black holes in matrix theory. 2., J. High Energy Phys. 01 (1998) 008.

[15] I. R. Klebanov and L. Susskind, Schwarzschild black holes in various dimensions from matrix theory, Phys. Lett. B 416, 62 (1998).

[16] E. Halyo, Six-dimensional Schwarzschild black holes in M (atrix) theory, arXiv:hep-th/9709225.

[17] G. T. Horowitz and E. J. Martinec, Comments on black holes in matrix theory, Phys. Rev. D 57, 4935 (1998).

[18] D. N. Kabat and W. Taylor, Spherical membranes in matrix theory, Adv. Theor. Math. Phys. 2, 181 (1998).

[19] J. M. Maldacena, The Large N limit of superconformal field theories and supergravity, Int. J. Theor. Phys. 38, 1113 (1999); Adv. Theor. Math. Phys. 2, 231 (1998)

[20] S. S. Gubser, I. R. Klebanov, and A. M. Polyakov, Gauge theory correlators from noncritical string theory, Phys. Lett. B 428, 105 (1998).

[21] E. Witten, Anti-de Sitter space and holography, Adv. Theor. Math. Phys. 2, 253 (1998).

[22] N. Itzhaki, J. M. Maldacena, J. Sonnenschein, and S. Yankielowicz, Supergravity and the large N limit of theories with sixteen supercharges, Phys. Rev. D 58, 046004 (1998).

[23] K. N. Anagnostopoulos, M. Hanada, J. Nishimura, and S. Takeuchi, Monte Carlo Studies of Supersymmetric Matrix Quantum Mechanics with Sixteen Supercharges at Finite Temperature, Phys. Rev. Lett. 100, 021601 (2008).

[24] S. Catterall and T. Wiseman, Black hole thermodynamics from simulations of lattice Yang-Mills theory, Phys. Rev. D 78, 041502 (2008).

[25] M. Hanada, Y. Hyakutake, J. Nishimura, and S. Takeuchi, Higher Derivative Corrections to Black Hole Thermodynamics from Supersymmetric Matrix Quantum Mechanics, Phys. Rev. Lett. 102, 191602 (2009).

[26] M. Hanada, Y. Hyakutake, G. Ishiki, and J. Nishimura, Holographic description of quantum black hole on a computer, Science 344, 882 (2014).

[27] D. Kadoh and S. Kamata, Gauge/gravity duality and lattice simulations of one dimensional SYM with sixteen supercharges, arXiv:1503.08499.

[28] M. Hanada, Y. Hyakutake, G. Ishiki, and J. Nishimura, Numerical tests of the gauge/gravity duality conjecture for D0-branes at finite temperature and finite N, Phys. Rev. D 94, 086010 (2016).

[29] E. Berkowitz, E. Rinaldi, M. Hanada, G. Ishiki, S. Shimasaki, and P. Vranas, Precision lattice test of the gauge/gravity duality at large- $N$, Phys. Rev. D 94, 094501 (2016).
[30] E. Rinaldi, E. Berkowitz, M. Hanada, J. Maltz, and P. Vranas, Toward holographic reconstruction of bulk geometry from lattice simulations, J. High Energy Phys. 02 (2018) 042.

[31] Y. Hyakutake, Quantum aspects of black objects in string theory, J. High Energy Phys. 01 (2017) 066.

[32] N. Kim, More on membranes in matrix theory, Phys. Rev. D 59, 067901 (1999).

[33] Y. Hyakutake, Torus-like dielectric D2-brane, J. High Energy Phys. 05 (2001) 013.

[34] J. Madore, The fuzzy sphere, Classical Quantum Gravity 9 , 69 (1992).

[35] P. A. Collins and R. W. Tucker, Classical and quantum mechanics of free relativistic membranes, Nucl. Phys. B112, 150 (1976).

[36] I. Chepelev and A. A. Tseytlin, Long distance interactions of branes: Correspondence between supergravity and super-Yang-Mills descriptions, Nucl. Phys. B515, 73 (1998).

[37] D. Ida, No Black Hole Theorem in Three-Dimensional Gravity, Phys. Rev. Lett. 85, 3758 (2000).

[38] S. S. Gubser, I. R. Klebanov, and A. A. Tseytlin, Coupling constant dependence in the thermodynamics of $N=4$ supersymmetric Yang-Mills theory, Nucl. Phys. B534, 202 (1998).

[39] Y. Hyakutake, Quantum near-horizon geometry of a black 0-brane, Prog. Theor. Exp. Phys. 2014, 033B04 (2014).

[40] N. Acharyya, N. Chandra, and S. Vaidya, Quantum entropy for the fuzzy sphere and its monopoles, J. High Energy Phys. 11 (2014) 078.

[41] S. D. Mathur, The Fuzzball proposal for black holes: An elementary review, Fortsch. Phys. 53, 793 (2005).

[42] H. Shimada, Membrane topology and matrix regularization, Nucl. Phys. B685, 297 (2004).

[43] H. Kawai, Y. Matsuo, and Y. Yokokura, A self-consistent model of the black hole evaporation, Int. J. Mod. Phys. A 28, 1350050 (2013).

[44] P. M. Ho, The absence of horizon in black-hole formation, Nucl. Phys. B909, 394 (2016).

[45] Y. Sekino and L. Susskind, Fast scramblers, J. High Energy Phys. 10 (2008) 065.

[46] P. Riggins and V. Sahakian, Black hole thermalization, D0 brane dynamics, and emergent spacetime, Phys. Rev. D 86, 046005 (2012).

[47] L. Brady and V. Sahakian, Scrambling with matrix black holes, Phys. Rev. D 88, 046003 (2013).

[48] E. Berkowitz, M. Hanada, and J. Maltz, Chaos in matrix models and black hole evaporation, Phys. Rev. D 94, 126009 (2016). 